\title{
Challenges in Membrane Process for Gas Separation from Natural Gas
}

\author{
K. Farahdila ${ }^{\mathrm{a}, b^{*}}$, P. S. Goh ${ }^{\mathrm{b}}$, A. F. Ismail ${ }^{\mathrm{b}}$, N. F. W. M. Wan ${ }^{\mathrm{a}}$, H. M. H. Mohd ${ }^{\mathrm{a}}$, \\ W. K. Soh ${ }^{\mathrm{a}} \&$ S. Y. Yeo
}

${ }^{a}$ Gas Sustainability Technology (GaSTech) R\&D Department, Group Research \& Technology (GR\&T), Project Delivery \& Technology (PD\&T), PETRONAS Research Sdn Bhd, Block E, Lot 3288 \& 3289, Off Jalan Ayer Itam, Kawasan Institusi Bangi, 43000 Kajang, Selangor, Malaysia

${ }^{\mathrm{b}}$ Advanced Membrane Technology (AMTEC), Universiti Teknologi Malaysia, 81310, UTM Johor Bahru, Johor, Malaysia

Submitted: 24/5/2021. Revised edition: 15/6/2021. Accepted: 15/6/2021. Available online: 15/7/2021

\begin{abstract}
Membrane technology is cost effective solution for $\mathrm{CO}_{2}$ removal from natural gas. However, there is challenges during its application depending on the polymer material characteristic. Understanding on the polymer fundamental and transport properties, will enable proper design of pre-treatment and operating conditions that suits its capability envelope. Diffusivity selective membrane favors high pressure and high temperature conditions and vice versa for solubility selective polymer. On top of that, the robustness and durability of the resultant membrane, need to be evaluated with multicomponent mixture to understand the effect of competitive sorption, plasticization and aging phenomena that will seriously impacting the membrane performance during its application.
\end{abstract}

Keywords: $\mathrm{CO}_{2}$ removal, mixed gas, plasticization, contaminants, aging

\subsection{INTRODUCTION}

Membrane is one of the technology of choice for $\mathrm{CO}_{2}$ removal from high contaminated gas fields especially for offshore application due to its cost effectiveness. Lots of polymers have been studied as membrane materials for $\mathrm{CO}_{2} / \mathrm{CH}_{4}$ gas separations, but small amounts have been commercialized for natural gas separation due to the effects of physical aging and plasticization (Low et al., 2018). The most commonly used material is cellulose based due to its robustness and stable performance despite lower selectivity. Other polymers that also have some recorded application are polysulfone, polyimide, and perfluoro polymers (Lokhandwala and Baker, 2008).

However, the contaminants present in the natural gas can be problematic to membrane polymeric material hence the understanding on the impact for each of the contaminants to the polymeric material of choice is very crucial. This is to ensure proper design of the pretreatment and also the right process conditions can be set for the system to ensure the membrane separation system reliability for long term operation. The biggest challenges are to maintain membrane stability throughout the lifespan with high permeability and selectivity. The stability of the membrane is very much affected by the 
competitive sorption, plasticization and aging phenomena.

Objective of the paper is to review and highlight on the importance impact of plasticization and aging to the membrane performance due to the present of contaminants in the natural gas. The prediction is to be done starting from modelling and to be validated by the experimental studies.

\subsection{MEMBRANE TRANSPORT}

There are five (5) mechanism of transport in gas separation mechanism. Among all, only solution diffusion is applied for dense membrane and thin skin selective layer (skin thickness between 0.1 to $1 \mu \mathrm{m}$ ) asymmetric glassy gas separation membrane (Scholes, Kentish, and Stevens, 2009). The asymmetric structure has the advantages of not only providing mechanical strength to the denseselective layer but also reducing the transport resistance across the membrane (Yong et al., 2018). This model postulated that permanent pores is not available in the membrane skin, hence separation happen due to variation of sorption and mobility of the gases via the polymer material (Galizia et al., 2017). Glassy membranes is use below its glass transition temperature hence the polymer chains are improperly packed, leading to space in between them which been called as fractional free volume (FFV) (Scholes, Kentish, and Stevens, 2009).

Diffusion happens in order to attain equilibrium, from higher concentration to lower and this lead to system entropy increases. Factors such as density, nature of polymer either rubbery or glassy, existence of free volume and many others affect the membrane transport (Favvas et al., 2017), (Freeman and Pinnau, 2004). The solutiondiffusion depends on the physicalchemical interaction between the polymer and the penetrant gases via solubility and diffusivity. The thermodynamic differences between the two membrane sides serve as the driving force. In order to separate a component in a particular feed mixture, the polarity of one component must be close to the polarity of the membrane. Polarity is one of the polymer properties that contribute considerably to the membrane separation performance specifically via solubility factor which is very dependent on the nature of the polymer (Scholes, Kentish, and Stevens, 2009).

The feed side gas is sorbed on the membrane surface that have high partial pressure, diffuses through and exits from the other side of the membrane that have low partial pressure and this happening in the polymer matrix free-volume elements ( 0.2 to $0.5 \mathrm{~nm}$ in size) that continuously appear and disappear as a result of thermal motion (Iarikov and Ted Oyama, 2011).

Pure gas permeability $(\mathrm{P})$ in the steady-state condition is given as:

$$
P_{A}=D_{A} \times S_{A} \quad \text { Equation } 1
$$

With $\mathrm{D}$ is the average effective diffusion coefficient, $\mathrm{S}$ is the solubility coefficient and $\mathrm{P}$ is stated in Barrer. For asymmetric and composite membranes, since it is difficult to measure the thickness of the thin skin layer, the permeance is used which equivalent to the permeability over thickness of dense layer and is stated in GPU. 


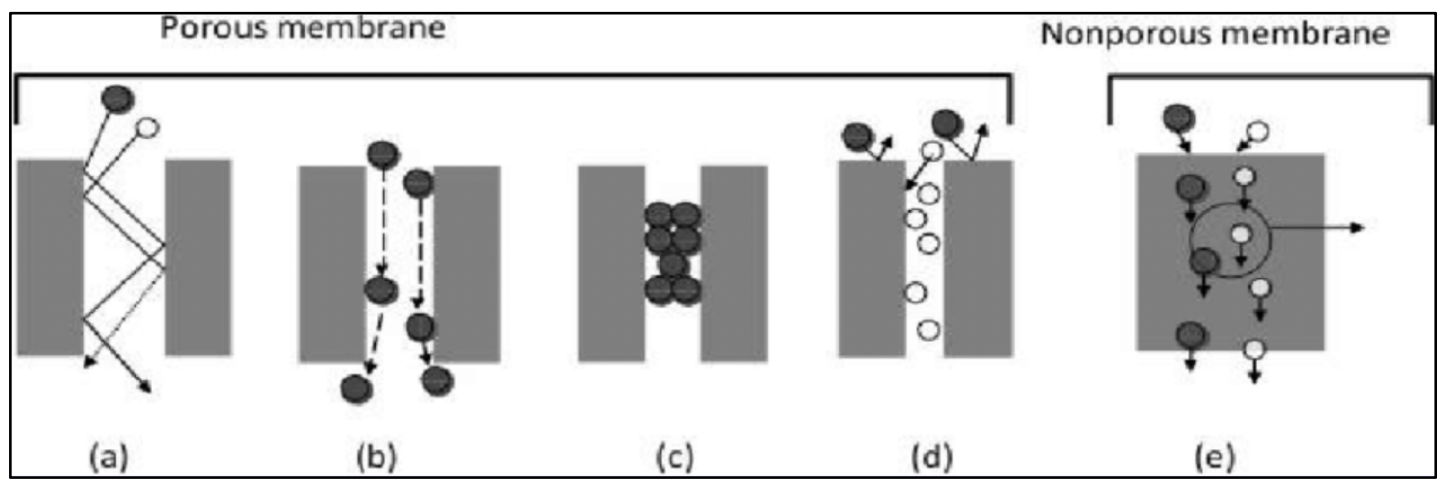

Figure 1 Schematic of membrane gas separation mechanism, (a) Knudsen- flow separation, (b) surface-diffusion, (c) capillary condensation, (d) molecular-sieving separation, and (e) solution-diffusion mechanism (Afzali, Maghsoodlou, and Noroozi, 2015)

Other than solution diffusion, there are many others transport mechanism for gas separation membrane as illustrates in Figure 1. Knudsen diffusion occurs when the pore size is smaller than the mean free path of molecules hence causing more collision with wall of the pore than with penetrants. For the case of pore size is roughly alike with gas size, molecular sieving mechanism happens since bigger penetrants cannot flow through it. Hagen-Poiseuille in the other hand, take places when the pore size is larger than the mean free path of the gases where the transport occur by the fluid bulk flow. Another one is surface diffusion of porous membranes which related to polarity between polymer material and the gases. Lastly, capillary condensation happening due to low vapour pressures that result in partial condensation thus, the condensed component diffuses more faster through the pore, causing the separation to happen (Scholes, Kentish, and Stevens, 2009).

Membrane performance is important in term of its selectivity value which determines the product purity, separation efficiency and hydrocarbon loss while permeance will determines the productivity which will relates to membrane area and footprint requirement. The trade off between permeability and selectivity in conventional membrane polymers was first evidence by Robeson in 1991 and then updated in 2008 illustrating the progress achieved by polymer science for the main gas separations (Bernardo and Clarizia, 2013). Significant shifts in the front factor are primarily due to recent data on perfluorinated, thermally rearranged, modified polyimide and super glassy polymers that did not exist in 1991 (Sanders et al., 2013). According to Freeman's theory, the polymer chain backbone stiffness is crucial on top of the availability of micropores in order to improve high membrane productivity and purity (Favvas et al., 2017).

\subsection{THEORY}

The ideal selectivity is the ratio of the two gases membrane permeabilities under the same conditions:

$$
\boldsymbol{\alpha}_{\mathrm{A} / \mathrm{B}}=\frac{\mathrm{P}_{\mathrm{A}}}{\mathrm{P}_{\mathrm{B}}}=\left(\frac{\mathrm{D}_{\mathrm{A}}}{\mathrm{D}_{\mathrm{B}}}\right) \times\left(\frac{\mathrm{S}_{\mathrm{A}}}{\mathrm{S}_{\mathrm{B}}}\right) \quad \text { Equation } 2
$$

Wher $D_{A} / D_{B}$ is the diffusivity selectivity and $S_{A} / S_{B}$ is the solubility selectivity. Component A is the more permeable species. 


\subsection{Fick's Law - Diffusion}

Steady state diffusion is stated by the Fick's first law ( (Wypych 2017):

$$
J=-D \frac{\partial c}{\partial x}
$$

Equation 3

With, $\mathrm{J}$ as penetrant flux, $\mathrm{D}$ is diffusion coefficient, $\mathrm{c}$ is penetrant concentration and $\mathrm{x}$ is penetration depth.

The Fick's second law of diffusion (non-steady state diffusion) is:

$$
\frac{\partial c}{\partial t}=-D \frac{\partial^{2} c}{\partial x^{2}}
$$

Equation 4

with $t$ represents time and any second Fick's law deviation is referred to nonFickean behavior.

\subsection{Henry's Law - Solubility}

Gas solubility within the rubbery polymer matrix which are in a hypothetical thermodynamic equilibrium liquid state (Kanehashi and Nagai, 2005) follows Henry's Law and is linearly proportional to the partial pressure, or fugacity, $f$ (Scholes, Kentish, and Stevens, 2009):

$$
C_{D}=K_{D} \mathbf{f}
$$

Equation 5

Where $C_{D}$ is the concentration of gas in the polymer matrix and is proportional through the Henry's Law constant $\left(\mathrm{K}_{\mathrm{D}}\right)$. The total concentration of absorbed gas within a glassy membrane (C) can be described by:

$$
\mathrm{C}=\mathrm{C}_{\mathrm{D}}+\mathrm{C}_{\mathrm{H}} \quad \text { Equation } 6
$$

where $\mathrm{C}_{\mathrm{H}}$ is the standard Langmuir adsorption relationship. Glassy polymer is typically assumed to be in nonequilibrium within hypothetical liquid and solid state (Kanehashi and Nagai, 2005).

\subsection{Arrhenius Equation - Diffusion as a Function of Temperature}

The connection among the polymeric membrane gas diffusion coefficient with temperature is given by (Adewole and Sultan, 2019):

$$
\mathbf{D}_{\mathrm{A}}=\mathbf{D}_{\mathrm{Ao}} \exp \left[\frac{-\mathrm{E}_{\mathrm{D}}}{\mathrm{RT}}\right] \quad \text { Equation } 7
$$

where $\mathrm{D}_{\mathrm{A}}$ represents the diffusion coefficient $\left(\mathrm{cm}^{2} / \mathrm{s}\right)$ of gas $\mathrm{A} . \mathrm{R}$ is the universal gas constant $(8.31 \mathrm{~J} / \mathrm{mol})$, $\mathrm{T}$ is the temperature (in $K$ ), $E_{D}$ is the activation energy of diffusion (in $\mathrm{kJ} / \mathrm{mol}$ ) and $\mathrm{D}_{\mathrm{A} 0}$ is the pre-exponential factor.

\subsection{Van't Hoff Equation - Solubility as a Function of Temperature}

Solubility relationship with temperature is given by (Adewole and Sultan, 2019):

$$
\mathbf{S}_{\mathrm{A}}=\mathbf{S}_{\mathrm{Ao}} \exp \left[\frac{-\Delta \mathrm{H}_{\mathrm{s}}}{\mathrm{RT}}\right] \quad \text { Equation } 8
$$

$\mathrm{S}_{\mathrm{A} 0}$ is the pre-exponential factor and $\Delta \mathrm{H}_{\mathrm{S}}$ enthaphy is gas A solution. The gas permeability is:

$$
\begin{aligned}
& \mathbf{P}_{A}=\mathbf{P}_{\text {Ao }} \exp \left[\frac{-E_{p}}{R T}\right] \quad \text { Equation } 9 \\
& \mathbf{E}_{\mathbf{P}}=\mathbf{E}_{\mathbf{D}}+\Delta \mathbf{H}_{\mathbf{S}}
\end{aligned}
$$

$\mathrm{P}_{\mathrm{A} 0}$ and $\mathrm{E}_{\mathrm{P}}$ are the pre-exponential factor, and the activation energy of permeation, respectively.

\subsection{Dual-mode Sorption Model}

Dual-mode model and partial/total immobilization models equation for gas transport behavior in glassy polymers below glass transition temperature $(\mathrm{Tg})$ is (Adewole and Sultan, 2019):

$$
\mathbf{S}=\mathbf{k}_{\mathbf{D}}+\frac{\mathrm{FC}_{\mathrm{H}}^{\prime} \mathbf{b}}{1+\mathbf{b}_{\mathbf{p}}}
$$

Equation 10 
where $\mathrm{k}_{\mathrm{D}}$, is Henry's law constant, $\mathrm{C}^{\prime} \mathrm{H}$, is Langmuir capacity constant, $b$ is Langmuir affinity constant and $F$ is set to 1 for total immobilization model,. Components having solubility parameters close to each other is miscible to each other (Wypych, 2017).

The permeability is then (Adewole and Sultan, 2019):

$$
\mathbf{P}=\mathbf{k}_{\mathbf{D}} \mathbf{D}_{\mathbf{D}}+\frac{\mathbf{C}_{\mathrm{H}}^{\prime} \mathbf{b D}_{\mathrm{H}}}{\mathbf{1}+\mathbf{b}_{\mathbf{p}_{2}}} \quad \text { Equation } 11
$$

where the Henry diffusion coefficient is $\mathrm{D}_{\mathrm{D}}, p$ is pressure and $\mathrm{D}_{\mathrm{H}}$ is Langmuir diffusion coefficient.

The dual-mode sorption parameters relate to several factors, namely the glass transition temperature, fractional free volume and gas permeability which is the diffusivity and solubility. The free volume is related to non-equilibrium structure exist in polymer formed during the membrane fabrication. Referring to Figure 2, it can be seen that as the temperature is increased, the excess free volume which is in the non-equilibrium state increases, as the the $\mathrm{Tg}$ increases. The higher the free volume, the higher the gas solubility for the particular polymer and the higher $\mathrm{Tg}$, the solubility of condensable gas also increased exponentially (Kanehashi and Nagai, 2005).

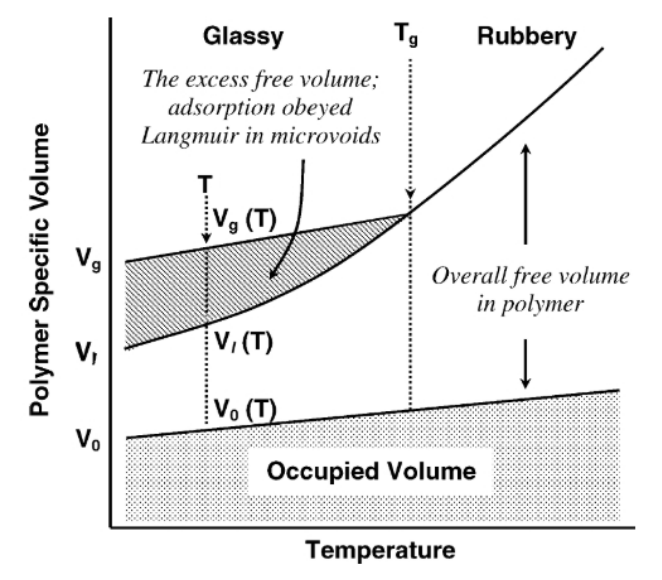

Figure 2 Polymer specific volume versus temperature in amorphous polymer (Kanehashi and Nagai, 2005)
Kentish and co-worker has detected that at low vapour activity, dual mode sorption is occurred where hydrocarbons is sorpted in polymer matrix. At more than 0.5 vapour activity, higher permeability has been found to be related with dilation and plasticisation of the polymer. Plasticisation has been found to happen at the particular palsticizer amount for specific polymer. The reduction in gas permeability at first might be partially due to "pore blocking", "competitive sorption" or "anti-plasticisation" phenomenon where the free space been occupied by the hydrocarbon and aromatic such as toluene which obstruct the flow path. At vapour pressure $>0.035$ bar, the increase in permeability occurred very rapidly due to the toluene-induced plasticisation. However, the toluene gas has no impact to the $\mathrm{CO}_{2} / \mathrm{CH}_{4}$ selectivity of the membrane (Lu et al., 2018).

The effects of plasticization are also proportional to the membrane thickness. As the thin skin layer decrease, the $\mathrm{CO}_{2}$ plasticization pressure also reduce to lower pressures such as for composite membranes with polyimide top layers, as illustrated in Figure 3 (Visser 2006).

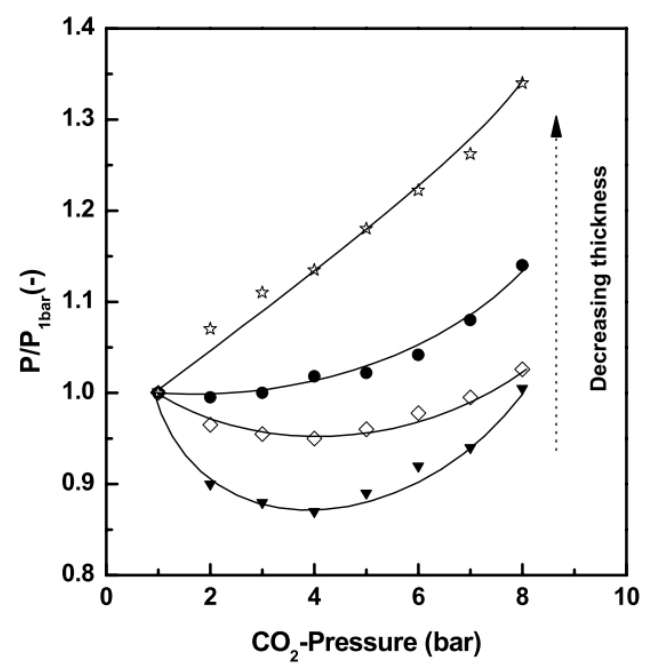

Figure $3 \mathrm{CO}_{2}$-permeance, normalized for the initial value at $p=1 \mathrm{bar}$, as a function of pressure for composite membranes with varying polyimide top layer thicknesses (Visser, 2006) 
Referring to the Figure 4, there is certain type of membrane material and certain membrane characteristic that will determine the permeation trending and whether it follows dual mode sorption theory. It can be seen that only Type 1 and II follow the reduction and increasing trend indicating the saturation and plasticization, while Type III do not have site saturation mechanism. Interestingly, cellulose acetate the commercial gas separation membrane for $\mathrm{CO}_{2}$ removal follows Type III while polysulfone, polycarbonate and polyimide follow Type 1 and II, indicating of higher $\mathrm{CO}_{2}$ plasticization pressure than cellulose acetate (Ismail and Lorna, 2002).

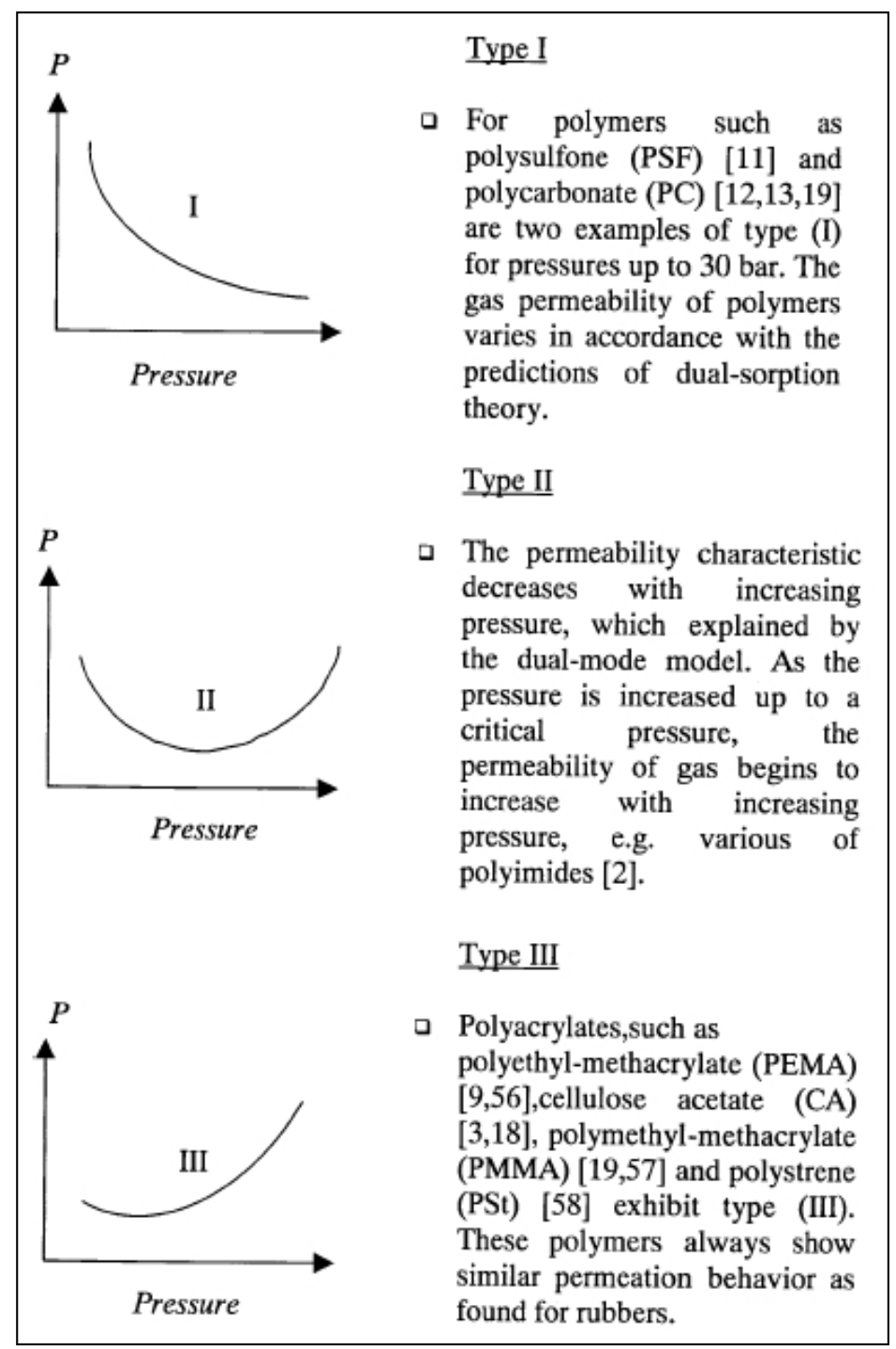

Figure 4 Representation permeabilities $(\mathrm{P})$ of several glassy polymers to $\mathrm{CO}_{2}$ (Ismail and Lorna, 2002) 


\subsection{Flory-Higgins (FH) \& Guggenheim Andersonde Boer (GAB) Model}

Advanced dual mode sorption models can be found such as the FH and GAB model (Lu et al., 2018). FH theory explains that both the enthalpy and entropy influence the polymer and plasticizer as per below (Wypych, 2017):

$$
\begin{aligned}
& \frac{\Delta G}{R T}=x_{1} \operatorname{In} \phi_{1}+x_{2} \operatorname{In} \phi_{2}+ \\
& \chi_{1} \phi_{1} \phi_{2}\left(x_{1}+x_{2} \frac{V_{2}}{V_{1}}\right.
\end{aligned} \quad \text { Equation } 12
$$

$\Delta \mathrm{G}$ is Gibbs free energy of mixing, $\mathrm{x}_{1}$ and $\Phi_{1}$ is mole and volume fraction of plasticizer whereas $\mathrm{x}_{2}$ and $\Phi_{2}$ for the polymer, $\chi 1$ is Huggins interaction which is dimensionless semi-empirical constant.

Several works to discover plasticization modeling related to higher permeability as a function of pressure has been done (Khademian et al., 2020; Lock et al., 2017; Miandoab, Kentish, and Scholes, 2021) Nevertheless, the correlation between the polymer material features with "plasticization pressure" are still non conclusive (Minelli et al., 2019). Kentish et al. tried to model the plasticization sorption isotherms using advanced dual mode sorption models such as the $\mathrm{FH}$ and GAB model but both is not suited and they proposed that more detail study need to be conducted with multiple parameters to predict the plasticization phenomenon (Lu et al., 2018).

The simplistic membrane model that does not account for such complex effects can deviate more than $20 \%$ from a more rigorous approach (Miandoab, Kentish, and Scholes, 2021).

\subsection{Free Volume Equation}

Free volume is (Alentiev and Yampolskii, 2000):
$D_{i}=F_{i} \exp \left(-\frac{V_{i}^{*}}{V_{f}}\right) \quad$ Equation 13

Where, $D_{i}$ is the diffussion coefficient, $V_{i}^{*}$ and $F_{i}$ are gas and gas/polymer system constant. Enhancement been done later, to consider the free volume overlapping by introducing $1>\gamma>0$ parameter and assumed $F_{i}$ is not related to temperature :

$$
D_{i}=F_{i} \exp \left(\frac{-\gamma V_{i}^{*}}{V_{f}}\right) \quad \text { Equation } 14
$$

Generally, gas solubility increases with increasing condensability (i.e. higher critical temperature or higher normal boiling point) and it has more favorable interactions with the polymer. In the other hand, higher gas diffusivity is achieved by decreasing penetrant size, increasing FFV (fractional free volume) and polymer chain flexibility. $\mathrm{CO}_{2}$ have higher solubility than light gases due to its higher condensability in addition to smaller molecular size that favors its diffusion. Polymer materials with high diffusivity selectivity have been largely explored for $\mathrm{CO}_{2} / \mathrm{CH}_{4}$ separation using high $\mathrm{Tg}$ and rigid structure while high $\mathrm{CO}_{2}$ permeability is achieved by sustaining or enhancing FFV. Another option is by using materials with higher solubility selectivity which is worth to explore since the diffusion coefficients are more sensitive than solubility when aging and densification set in. This can be done using ether oxygens in ethylene oxide (EO) units to take advantage of polar groups attraction in polymers with $\mathrm{CO}_{2}$. This solubility selectivity is actually been used by the commercial cellulose based membrane (Lin and Freeman, 2005). The polymer material must also be evaluated with condensable gases other than $\mathrm{CO}_{2}$ that may present in the natural gas, since these bigger condensable molecules such as heavy hydrocarbons can severely impacted the membrane separation ability. Therefore, 
polymer materials for the use of gas separation have three categories related to its dominant factor of diffusivity selectivity, solubility selectivity, or both (Freeman and Pinnau, 2004).

\subsection{Partial Immobilization Model}

In partial immobilization model, it was assumed that not all gas molecules been sorbed are mobile and contribute to the gas transport which related to the gas phase fugacity on both of the products namely retentate and permeate. This model also includes the term to account for competitive sorption (Miandoab, Kentish, and Scholes, 2021).

$$
C_{i}^{k}=\left(\boldsymbol{k}_{D, i}+\frac{F_{i} \boldsymbol{C}_{H, i}^{\prime} \boldsymbol{b}_{i}}{1+\sum_{j} \boldsymbol{b}_{j} f_{j}^{k}}\right) \boldsymbol{f}_{i}^{\boldsymbol{k}} \quad \text { Equation } 15
$$

Where $j$ is the components in the gas, $F$ is the mobile portion of the adsorbed molecules in the Langmuir region, $k_{D}$ is the Henry's law solubility coefficient, $C_{H}^{\prime}$ is the Langmuir's sites saturation constant and $b$ is an affinity constant. The solubility coefficient, saturation and affinity constant are determined by fitting the classical dual mode sorption model to experimental single gas sorption. However, the use of single gas data for mixed gas prediction is not accurate. $\sum_{j} b_{j} f_{j}$ is added to the model to cater for competitive sorption.

To take into account of plasticization, Equation 16 and Equation 17 below are to be included in Fick's first law (Equation 3) and integrate it from high pressure retentate to low pressure permeate. The final equation is illustrate in Figure 5. $D_{o}$ is the concentration independent diffusivity at infinite dilution $(\mathrm{C} \rightarrow 0) . \boldsymbol{\beta}_{\boldsymbol{A}}$ is the plasticization factor if $\mathrm{A}$ and it is rely on temperature and interactions between component and polymer. $\boldsymbol{\beta}_{\boldsymbol{A}-\boldsymbol{B}}$ is to consider the effect of membrane plasticization due to A on the diffusivity of B (Miandoab, Kentish, and Scholes, 2021).

$$
\begin{array}{ll}
D_{A}^{k}=D_{o A} \exp \left(\beta_{A} C_{A}^{k}\right) & \text { Equation } 16 \\
D_{B}^{k}=D_{o B} \exp \left(\beta_{A-B} C_{A}^{k}\right) & \text { Equation } 17
\end{array}
$$

$$
P_{B}^{k}=\frac{D_{0 B}}{f_{B, R}^{k}-f_{B, P}^{k}} \times \exp \left\{\beta_{A-B}\left(k_{D, A}+\frac{F_{A} C_{H, A}^{\prime} b_{A}}{1+\sum_{j} b_{j} f_{j, R}^{k}}\right) f_{A, R}^{k}\right\} \times\left[\left(k_{D, B}+\frac{F_{B} C_{H, B}^{\prime} b_{B}}{1+\sum_{j} b_{j} f_{j, R}^{k}}\right) f_{B, R}^{k}-\left(k_{D, A}+\frac{F_{B} C_{H, A}^{\prime} b_{A}}{1+\sum_{j} b_{j} f_{j, P}^{k}}\right) f_{B, P}^{k}\right]
$$

Figure 5 Fugacity-dependent permeabilities of A and B under dry conditions

\subsection{COMPETITIVE SORPTION}

Competition effect from other gases can only be seen when multicomponent testing been done, which will reduce the permeability of the desired component, $\mathrm{CO}_{2}$. The competition effect is said to severely impacted the diffusivity selective membrane, with lesser impact to the solubility dominant membrane leveraging on its polarity, in addition to operating condition that favor it. The solubility is favors during low operating temperature but reduced at high operating pressure and temperature application. Hence the tuning of operating pressure and temperature is important to leverage on the polymer material characteristics. Despite that, the polymer material must also robust to other plasticizers present in the feed that might sorp through the polymer matrix, blocked other penetrants and swell the membrane. 


\subsection{PLASTICIZATION AND AGING}

Plasticization is the major issue in gas separation membrane operation (Lokhandwala and Baker, 2008) and has been identified to be correlated to polymer chain softening, resulting in higher mobility. Dissolution of plasticizers affect material thermal and mechanical properties such as $\mathrm{Tg}$ and rigidity reduction, increased in elongation at break and impact strength (Minelli et al., 2019). Its deteriorate the membrane performance till the membrane loss its separation ability (Houben, Borneman, and Nijmeijer, 2020).

Membrane aging set in during the application of the membrane leads to densification, reduction in FFV hence reduced the permeability while enhancing the selectivity. The permeability reduction will be measured till it cannot meet the product flow target, hence certain portion of the membrane will need to be replaced (Xia, Chung, and Paul, 2014). The typical membrane life will be minimum 3 years and this is crucial for the design of membrane system to ensure continous in meeting the product flowrate and specifications. Usually membrane spares is provided in the design, so that membrane can be changed out online without the need to shutdown the whole system.

Natural gas are comprised of $\mathrm{CO}_{2}$, $\mathrm{CH}_{4}, \mathrm{C}_{2}$ to $\mathrm{C}_{6}$ (after pre-treatment), aromatic hydrocarbons and many others minor contaminants. Roughly 1000 ppm of water and up to $500 \mathrm{ppm}$ of benzene, toluene, ethylbenzene and xylene (BTEX) present in the gas streams at the operating pressure of 20 to 60 bar, causing the membrane material to experience plasticization, competitive sorption, and aging (Iulianelli and Drioli, 2020). At the moment, cellulose based membrane still dominate for the use in natural gas processing despite its low selectivity and higher hydrocarbon loss. This is due to its polymer characteristic that is robust to heavy hydrocarbon and aromatic supported with comprehensive pre-treatment properly designed to cater for its limitations to water hence the application is only within the membrane specific capability envelope.

Although cellulose based membrane has the lowest $\mathrm{CO}_{2}$ plasticization pressure, that is not really the disadvantages as long it can be controlled and not impacted the overall polymer separation capability severely.

\section{$5.1 \mathrm{CO}_{2}$}

$\mathrm{CO}_{2}$ plasticization enhance the chain mobility leads to increase in $\mathrm{CO}_{2}$ diffusivity as a function of $\mathrm{CO}_{2}$ content and pressure. Solubility in the other hand, reduces as a function of $\mathrm{CO}_{2}$ pressure due to glassy state volume constraints. This opposite trends of solubility and diffusivity will determine the resultant permeability trend at the initial testing prior to plasticization point. Plasticization that is present during the membrane operation at high pressure can only dominate the effect to the permeability when it is adequately high as compared to the $\mathrm{CO}_{2}$ effects on solubility coefficient resulting to permeability increase instead of decreasing (Minelli et al., 2019). Operating temperature can play a role in increasing the solubility term at high pressure operation, where low temperature operation is favourable to boost the permeability by the solubility.

Membrane materials absorb 5 to 10 wt $\%$ of $\mathrm{CO}_{2}$ in the polymer at high pressure operation between 30 to 60 bar. The absorbed $\mathrm{CO}_{2}$ swells the cellulose acetate polymer and result in opposing way for the single and mixed gas membrane selectivities. Pure gas selectivity increases due to the increase of $\mathrm{CO}_{2}$ permeability with constant $\mathrm{CH}_{4}$ permeability but the mixed gas 
selectivity reduced with pressure due to $\mathrm{CO}_{2}$ swelling that increase the $\mathrm{CH}_{4}$ permeability more than its single gas value (Buonomenna 2017).

Conditioning the membrane at 30 bar pressure with $\mathrm{CO}_{2}$ gas result in permanent effects to the polymer chain. Glassy polymer features are influenced by the polymer chains that frozed below their glass transition point (lower mobility state). The $\mathrm{CO}_{2}$ that act as a plasticizer causing slight changed to the glassy state arrangement that is not recoverable after removal of $\mathrm{CO}_{2}$ (Minelli et al., 2019). This effect can be leverage to enhance the permeability or usually been called as good plasticization effect when the selectivity is not affected so much.

$\mathrm{CO}_{2}$ plasticization resistant is shown by the new superglassy polymer called PIM-1 up to 15 bar pressure for single $\mathrm{CO}_{2}$ gas test. $\mathrm{Up}$ to 30 bar pressure, PIM- 1/POSS (98:2) and (90:10) also show plasticization and aging resistant behaviour during the mixed gas tests with $2 \mathrm{wt} \%$ loading of POSS nanoparticles in PIM-1 displays the best membrane performance. The substantial improvement in diffusivity is assumed to be the main contributor of higher permeability while the POSS rigid cage help in improvement of membrane stiffness. The data shows that low loading POSS interrupt the polymer matrix packing and at higher concentration result in chain rigidification. This result can be a potential solution for improvement in polymeric membrane permeability, aging resistance and suppression of plasticization (Yong et al., 2015).

Nevertheless, the effect of plasticization for a particular polymer cannot be analysed using $\mathrm{CO}_{2}$ only for the natural gas processing application. There are lots of other contaminants that are present and will need to be evaluated. They might affect the membrane more severely than the small $\mathrm{CO}_{2}$ gases i.e., heavy hydrocarbon and aromatic that will make the membrane loss its separation ability.

\section{$5.2 \mathrm{H}_{2} \mathrm{O}$}

Other contaminants that presence in minor amount in the feed gas of membrane gas separation process may compete with $\mathrm{CO}_{2}$ to permeate hence decrease the $\mathrm{CO}_{2}$ permeability. The contaminants also can damage the membrane thus alter the separation efficiency negatively. The water permeability is generally higher than $\mathrm{CO}_{2}$ for polymeric membranes since it has a smaller Lennard-Jones kinetic diameter than $\mathrm{CO}_{2}$ (2.65 Angstrom versus 3.3 Angstrom), and can diffuses more rapidly. It also has higher critical temperature $(374 \mathrm{~K}$ vs $304 \mathrm{~K})$, more condensable, have higher Langmuir affinity constant resulting in higher solubility. Although its partial pressure is low, water will block the voids in the polymer matrix due to smaller kinetic diameter and higher affinity or condensability reducing the $\mathrm{CO}_{2}$ permeability (Scholes, Kentish, and Stevens, 2009). 


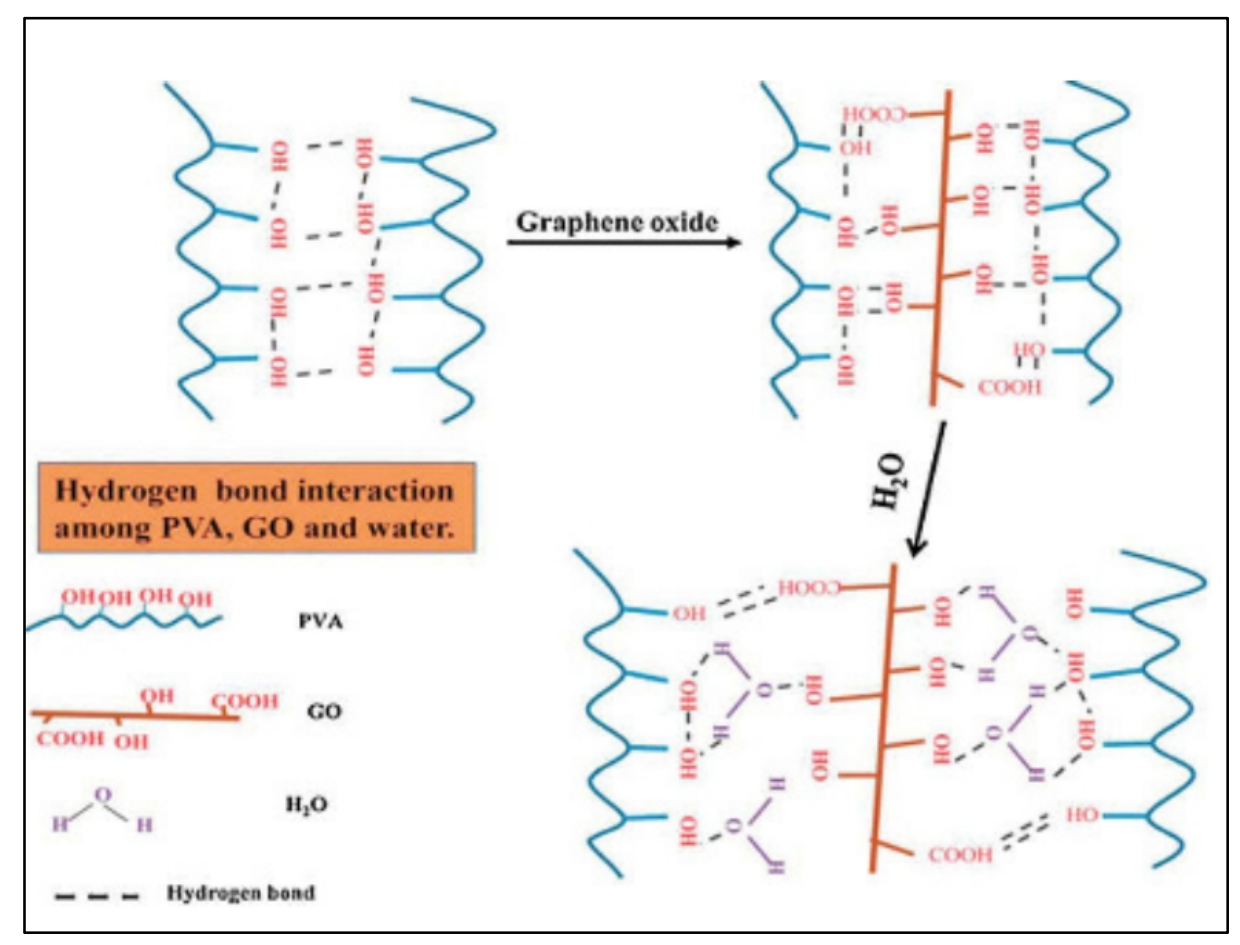

Figure 6 Poly(vinyl alcohol)/graphene oxide composite that has been plasticized by water molecule (Wypych, 2017)

Figure 5 illustrates the water (plasticizer) generate bridges between polymer matrix and reinforcement (graphene oxide), which enhance volume and elasticity. These bridges are formed by hydrogen bonding and the same mechanism can be applied to carbon nanotubes (Wypych, 2017).

As co-plasticizer, water changes the membrane properties (Wypych, 2017). Exposure to water showing significant permeability increases by $250 \%$, through polyethersulfone membrane and the permeability remains high irrespective of humidity concentration. Selectivity also been reduced by $25 \%$ due to water-induced plasticization. Cellulose acetate exposed to feed gas with water also showing the same impact of plasticization and competition. Firstly, at small concentration of $\mathrm{H}_{2} \mathrm{O}$, the permeability increases, revealing of plasticization phenomena. The permeability start to decreases at higher $0.85 \%$ humidity since cellulose acetate is a dry hydrophilic membrane that will be hydrated with the present of water causing it to swell. Moisture levels up to $20 \%$ relative humidity result to only a $20 \%$ reduction in permeability per month, but more than that result in very fast unrecoverable loss. This is hypothesized to be due to moisture condensation serve as pore blocking. Therefore, some will set the operating tempature at least $10{ }^{\circ} \mathrm{C}$ above the dew point of the feed gas mixture. This is to prevent the condensation impacts that can permanently alter the membrane structure even after the membrane been dried (Scholes, Kentish, and Stevens, 2009).

Usually, there will be dehydration unit upfront of membrane process system for water removal in order to prevent all these effects which is harmful for the membrane separation efficiency. 


\subsection{Hydrocarbon}

Incomplete reformation of fossil fuel leads to natural gas containing trace components of numerous paraffinic and aromatic hydrocarbons and the condensable hydrocarbons $\left(\mathrm{C}^{+}+\right)$that can modify the structure of polymeric membranes hence affecting their performance. The incorporation of toluene to a 6FDA- DMB polyimide leads to methane permeability enhancement, while no change occurs for $\mathrm{CO}_{2}$ which might be due to competition effect hence $\mathrm{CO}_{2} / \mathrm{CH}_{4}$ selectivity reduced drastically. Plasticization will normally increase the permeability of $\mathrm{CO}_{2}$ and $\mathrm{CH}_{4}$ while competitive sorption will decrease the $\mathrm{CO}_{2}$ permeability. Gases that exhibit higher sorption in polymers are more likely to cause plasticization (Scholes, Kentish, and Stevens, 2009). Typically, there will be adsorbent in place in the dehydration unit upfront of membrane process system for heavy hydrocrabon removal $\left(\mathrm{C}^{+}+\right)$in order to prevent all these effects which is damaging for the membrane separation performance.

\subsection{Liquid}

Petroleum based oil, paraffinic, naphthenic and aromatic oils are plasticizers (Wypych, 2017). Most of glassy polymer material are not robust to the condensing liquid which is generated when the gas been permeated. The liquid can fill the pore, swell the membrane, hinder the separation and deteriorate the membrane performance. Therefore, feed pre-heater will be placed in order to avoid condensation to happen. Joule Thompson (JT) cooling occur in the membrane during the $\mathrm{CO}_{2}$ removal, reduces the residue temperature. Heavy hydrocarbons which are retained and concentrated in the residue, after the $\mathrm{CO}_{2}$ been removed causes the phase envelope to shift to higher temperature.
These two effects will make the gas reach the saturation temperature of the hydrocarbon gas in residue and condensation start to occur. Temperature drop due to JT effect and pressure drop across the membrane cause the change in membrane permeance while condensation will affect the membrane material which will reduce the overall separation performance. In addition, there is the likelihood of condensation to happen at the permeate stream if the temperature is below the dew point, that will create an additional transfer layer for the gases to cross, hence impacting the membrane performance negatively and also problems to the permeate downstream processing. In addition, membrane sub-structure mechanical degradation can also happening due to capillary pressure effects resulting from condensed water evaporation (Scholes, Kentish, and Stevens, 2009), (Ahmad et al., 2013), (Lokhandwala and Baker, 2008).

In many cases polymers are contacted with liquids which penetrate its structure and plasticize it. Permeation of solvent through pervaporation membrane is a special case of such situation (Wypych, 2017) since it is been designed for gas liquid separation by using polymer material that can withstand the present of liquid.

\section{$5.5 \mathrm{H}_{2} \mathrm{~S}$}

$\mathrm{H}_{2} \mathrm{~S}$ is also plasticizer for the polymeric membranes. The effect on cellulose acetate for 2 weeks testing duration is examined. There is only little reduction happening within $5 \% \mathrm{CO}_{2}$ permeance with the presence of $\mathrm{H}_{2} \mathrm{~S}$ in the feed gas but the decrease is more severe up to $35 \%$ with the presence of water. This is hypothesized due to water competitive sorption effects and weak acidic degradation induced by $\mathrm{H}_{2} \mathrm{~S}$, which may impact the polymer chain negatively 
hence hampering the diffusion of gas (Scholes, Kentish, and Stevens, 2009).

$\mathrm{H}_{2} \mathrm{~S}$ has higher condensibility than $\mathrm{CH}_{4}(374 \mathrm{~K}$ vs $191 \mathrm{k}$, respectively) and $\mathrm{H}_{2} \mathrm{~S}$ also has nearly similar kinetic diameter with $\mathrm{CH}_{4}$ (3.6 vs 3.8 angstrom, respectively). Therefore, it is suitable to use rubbery polymers for $\mathrm{H}_{2} \mathrm{~S} / \mathrm{CH}_{4}$ separation which used solubility difference instead of kinetic difference. However, for smaller and more condensable $\mathrm{CO}_{2}$ versus $\mathrm{CH}_{4}(304 \mathrm{~K}$ vs $191 \mathrm{k}$, respectively), glassy polymers are more suitable by exploiting both sorption and diffusion parameters of $\mathrm{CO}_{2}$. Simultaneous $\mathrm{CO}_{2}$ and $\mathrm{H}_{2} \mathrm{~S}$ removal, possed a challenge, where $\mathrm{H}_{2} \mathrm{~S}$ permeability and $\mathrm{H}_{2} \mathrm{~S} / \mathrm{CH}_{4}$ selectivity of the non-crosslinked 6FDA-DAM/DABA membrane increases continuously as a function of pressure and vice versa for $\mathrm{CO}_{2} / \mathrm{CH}_{4}$ selectivity, which decreases. Glassy polyimides for $\mathrm{CO}_{2} \mathrm{CH}_{4}$ separation is quite promising but not for $\mathrm{H}_{2} \mathrm{~S} / \mathrm{CH}_{4}$ separation. Hence, the study for $\mathrm{H}_{2} \mathrm{~S} / \mathrm{CO}_{2} / \mathrm{CH}_{4}$ ternary gas separation is needed. $\mathrm{H}_{2} \mathrm{~S} / \mathrm{CH}_{4}$ show opposing trend to known plasticization effects where gas selectivity typically decreases with the increase of gas permeability, as observed in $\mathrm{CO}_{2} / \mathrm{CH}_{4}$. It is hypothesized that the conflicting effect of $\mathrm{CO}_{2} / \mathrm{CH}_{4}$ and $\mathrm{H}_{2} \mathrm{~S} / \mathrm{CH}_{4}$ separation plasticization effect is due to different dominant mechanisms for these two gas pairs where $\mathrm{CO}_{2}$ favors diffusion and $\mathrm{H}_{2} \mathrm{~S}$ favors solubility. $\mathrm{CH}_{4}$ permeation is inhibited by $\mathrm{H}_{2} \mathrm{~S}$ and $\mathrm{CO}_{2}$ leads to selectivity improvement. At the sametime, $\mathrm{CO}_{2}$ and $\mathrm{H}_{2} \mathrm{~S}$ permeabilities enhancement during the ternary gas permeation process in the 6FDA-based polyimide membranes is due to plasticization (Liu, Liu, Liu, et al., 2020) that swell the membrane slightly which is not big enough for $\mathrm{CH}_{4}$ to pass through. This is a good plasticization effect that enhance the permeability at certain period before reaching the point of selectivity reduction when the membrane been plasticized severely.

The above finding is supported by the study by Koros et al., where they used $20 \mathrm{~mol} \% \mathrm{H}_{2} \mathrm{~S}, 5 \mathrm{~mol} \% \mathrm{CO}_{2}, 3$ mol\% of $\mathrm{C}_{2} \mathrm{H}_{6}, 3 \mathrm{~mol} \%$ of $\mathrm{C}_{3} \mathrm{H}_{8}$, trace amount of toluene $(100-300 \mathrm{ppm})$ with $\mathrm{CH}_{4}$ comprising the rest of the feed tested with CTA hollow fiber provided by Cameron, a Schlumberger Company. The results showed unexpected increase in $\mathrm{H}_{2} \mathrm{~S} / \mathrm{CH}_{4}$ selectivity at $35{ }^{\circ} \mathrm{C}$ and 31.2 bar, which is contrary to traditional understanding of plasticization effect. $\mathrm{H}_{2} \mathrm{~S}$ permeance is $\sim 140 \mathrm{GPU}$ with $\mathrm{H}_{2} \mathrm{~S} / \mathrm{CH}_{4}$ selectivity of $\sim 28$ while $\mathrm{CO}_{2}$ permeance is $\sim 115 \mathrm{GPU}$ with $\mathrm{CO}_{2} / \mathrm{CH}_{4}$ selectivity of $\sim 22$. This finding allows the membrane to remove more $\mathrm{H}_{2} \mathrm{~S}$ than $\mathrm{CO}_{2}$ to assist in reducing final polishing to meet the pipeline specifications and demonstrates the simultaneous removal of $\mathrm{H}_{2} \mathrm{~S}$ and $\mathrm{CO}_{2}$ capability of the membrane. It shows that plasticization do promotes $\mathrm{H}_{2} \mathrm{~S} / \mathrm{CH}_{4}$ separation performance by providing more sorption capacity on the newly created free volume in the polymer (Liu, Liu, Morisato, et al., 2020).

\subsection{Mercury}

Highly toxic mercury posses corrosion issues especially in aluminium although they present in very small amount at part per billion (ppb) level. Due to its large kinetic diameter, it will be in retentate stream of membrane hence it will passed to the downstream processing plants. It is also likelihood of mercury accumulation across operating duration in the membrane voids over time that can hinder the gas transport since it has very high condensability factor as a result of high critical temperature (Scholes, Kentish, and Stevens, 2009). Therefore, it is advisable to install mercury removal unit prior to membrane process system. 


\subsection{BTEX}

BTEX (benzene, toluene, ethylbenzene and xylene) are condensable aromatic hydrocarbons exist in raw natural gas between 200 to $3000 \mathrm{ppm}$. It creates a substantial challenge (Lu et al., 2018) since they affect the polymeric membranes transport, by competing for sorption sites, hindering the gas transport and swell the polymer. The toluene and xylene can dilate the polymer and permeate thru the membrane together with methane (Lu et al., 2018). Toluene sorption rate is higher than xylene due to smaller kinetic diameter of toluene 5.85 Angstrom versus para-xylene 5.85 Angstrom and ortho and meta-xylene of 6.8 Angstrom eventhough the solubility of xylene is slightly higher than toluene by comparing its critical temperatures of 411- $417 \mathrm{~K}$ versus $384 \mathrm{~K}$ for toluene (Galizia et al., 2017).

Toluene is recorded to plasticize polyimides and perfluoropolymers by reducing both $\mathrm{CO}_{2}$ permeability also $\mathrm{CO}_{2} / \mathrm{CH}_{4}$ selectivity. Cellulose acetate membranes shows declining of $\mathrm{H}_{2}$ and $\mathrm{CH}_{4}$ permeabilities by 10 to $15 \%$ with $10 \%$ increase of $\mathrm{H}_{2} / \mathrm{CH}_{4}$ selectivity with the presence of toluene (up to $2.5 \mathrm{kPa}$ ) or benzene (up to $8.8 \mathrm{kPa}$ ). Cosmo Oil Refinery (Japan) that use cellulose acetate membrane for hydrogen separation, has reported stable operation for more than three years duration, eventhough the feed gas contains BTX aromatic $(1.8-5.2 \mathrm{kPa})$. The overall permeability reduction is only $20 \%$ throughout the operation period. Other study also reported that cellulose acetate membrane showing $10 \%$ decrease in $\mathrm{CO}_{2}$ and $\mathrm{CH}_{4}$ permeability with the presence of BTX $(5.4 \mathrm{kPa})$ in the binary feed gas of $6 \% \mathrm{CO}_{2}$ and $94 \% \mathrm{CH}_{4} .90 \%$ decline in gas permeabilities and 30 to $50 \%$ in selectivity for polyimide membrane with the present of BTX has been reported in the literature which is much more severe than cellulose acetate. Cellulose acetate membranes pure gas $\mathrm{CO}_{2} / \mathrm{CH}_{4}$ selectivity is 35 to 40 , binary gas at high pressure and $50{ }^{\circ} \mathrm{C}$ have a selectivity of 15 to 20 and this will further reduce to $10-14$ when tested with real natural gas feed with $\mathrm{C}_{2}$ to $\mathrm{C}_{6}$ hydrocarbons and BTEX in ppm level (Galizia et al., 2017).

The $\mathrm{CO}_{2}$ and BTEX plasticize the polymer hence reduce the $\mathrm{Tg}$ and transition to a swollen rubbery state happens at a lower temperature. It is also been hypothesized that there is a negative change in polymer crystallinity and increase in accessible free volume that decline the diffusivity selectivity (Lu et al., 2018).

Investigation been done on the influence of toluene on single and binary gas of $\mathrm{CO}_{2}$ and $\mathrm{CH}_{4}$ permeability in Thermally Rearranged polymer (TR450$30 \mathrm{~min}$ ). At a toluene activity of 0.2 , 93\% reduction in $\mathrm{CO}_{2}$ and $\mathrm{CH}_{4}$ permeability has been found as a result of competitive sorption and antiplasticization since toluene is much more condensable and fill in Langmuir sites preferably hence $\mathrm{CO}_{2}$ and $\mathrm{CH}_{4}$ solubility reduced. Large size of toluene hinders polymer matrix mobility and reduce diffusion (antiplasticization). These effects result in $8 \%$ increase in mixed $\mathrm{CO}_{2} / \mathrm{CH}_{4}$ selectivity at 0.2 toluene activity and $27 \%$ decrease in selectivity for the 0.7 toluene activity which caused by antiplasticization, plasticization and competitive sorption combined impact. Toluene vapor sorption experiments show that when Langmuir sites become completely saturated (at a toluene activity greater than 0.2 ), vapor sorption starts to occur in the Henry mode, which produces severe plasticization. However, this effects were largely recovered upon toluene removal from the feed (Galizia et al., 2017). 


\section{CONCLUSION}

As conclusion, the research in membrane material performance enhancement cannot be focused to the improvement in terms of permeability and selectivity only. However, the focus need to be done to find the balance between the performance with robustness and durability of the membrane when been applied at the real harsh condition of the natural gas feed. The superior performance might deteriorate instantly, if the material cannot withstand the contaminants and operating conditions impact such as plasticization and densification due to high operating pressure and aging phenomenon. The robustness to contaminants other that $\mathrm{CO}_{2}$ gas is crucial, since the polymer swelling due to heavies and aromatic which is bigger in size than $\mathrm{CO}_{2}$ and $\mathrm{CH}_{4}$ will make the membrane loss its separation ability. Plasticization of $\mathrm{CO}_{2}$ only, at certain condition can be leveraged to achieve higher permeability while the membrane need to be robust to the other plasticizers. On top of that, the plasticization impact of increasing the permeability will be lessen by the counter balance effect from the competitive sorption and aging which reduce the permeability.

\section{REFERENCES}

[1] Adewole, Jimoh K., and Abdullah S. Sultan. 2019. Polymeric Membranes for Natural Gas Processing: Polymer Synthesis and Membrane Gas Transport Properties. Functional Polymers, Polymers and Polymeric Composites.

[2] Afzali, Arezoo, Shima Maghsoodlou, and Babak Noroozi. 2015. Nanoporous Polymer/Carbon Nanotube
Membrane Filtration: The "HowTo" Guide to Computational Methods.

[3] Ahmad, Faizan, K. K. Lau, A. M. Shariff, and Yin Fong Yeong. 2013. Temperature and Pressure Dependence of Membrane Permeance and Its Effect on Process Economics of Hollow Fiber Gas Separation System. Journal of Membrane Science 430: 44-55. http://dx.doi.org/10.1016/j.memsc i.2012.11.070.

[4] Alentiev, A. Yu, and Yu, P. Yampolskii. 2000. Free Volume Model and Tradeoff Relations of Gas Permeability and Selectivity in Glassy Polymers. 165: 201-16.

[5] Bernardo, P, and G. Clarizia. 2013. 30 Years of Membrane Technology for Gas Separation. Chemical Engineering Transactions. 32: 1999-2004.

[6] Buonomenna, Maria G. 2017. Membrane Separation of $\mathrm{CO} 2$ from Natural Gas. Recent Patents on Materials Science. 10(1): 2649.

[7] Favvas, Evangelos P. et al. 2017. A Review of the Latest Development of Polyimide Based Membranes for $\mathrm{CO}_{2}$ Separations. Reactive and Functional Polymers. 120: 104-30.

[8] Freeman, Benny D., and Ingo Pinnau. 2004. Gas and Liquid Separations Using Membranes: An Overview. Americal Chemical Society. 1-23.

[9] Galizia, Michele et al. 2017. 50th Anniversary Perspective: Polymers and Mixed Matrix Membranes for Gas and Vapor Separation: A Review and Prospective Opportunities. Macromolecules. 50(20): 7809-43.

[10] Houben, H. J. M., Z. Borneman, and K. Nijmeijer. 2020. Plasticization Behavior of Crown- 
Ether Containing Polyimide Membranes for the Separation of CO2. Separation and Purification Technology. 117307.

[11] Iarikov, Dmitri D., and S. Ted Oyama. 2011. Membrane Science and Technology. Review of CO2/CH4 Separation Membranes.

[12] Ismail, A. F., and W. Lorna. 2002. Penetrant-Induced Plasticization Phenomenon in Glassy Polymers for Gas Separation Membrane. Separation and Purification Technology.

[13] Iulianelli, Adolfo, and Enrico Drioli. 2020. Membrane Engineering: Latest Advancements in Gas Separation and Pre- Treatment Processes, Petrochemical Industry and Re Fi Nery, and Future Perspectives in Emerging Applications. Fuel Processing Technology 206(March): 106464. https://doi.org/10.1016/j.fuproc. 2 020.106464.

[14] Kanehashi, Shinji, and Kazukiyo Nagai. 2005. Analysis of DualMode Model Parameters for Gas Sorption in Glassy Polymers. 253: 117-38.

[15] Khademian, Einallah, Mostafa Keshavarz Moraveji, Mitra Dadvar, and Hamidreza Sanaeepur. 2020. Plasticization Modeling in Cellulose Acetate / NaY Mixed Matrix Membranes Graphical Abstract Keywords. 6: 367-74.

[16] Lin, Haiqing, and Benny D Freeman. 2005. Materials Selection Guidelines for Membranes That Remove $\mathrm{CO}_{2}$ from Gas Mixtures. 739: 57-74.

[17] Liu, Yang, Zhongyun Liu, Atsushi Morisato, et al. 2020. Natural Gas Sweetening Using a Cellulose Triacetate Hollow Fiber Membrane Illustrating Controlled Plasticization Benefits. Journal of
Membrane

Science 601(December 2019): 117910.

[18] Liu, Yang, Zhongyun Liu, Gongping Liu, et al. 2020. Surprising Plasticization Bene Fi Ts in Natural Gas Upgrading Using Polyimide Membranes. Journal of Membrane Science. 593(June 2019): 117430.

[19] Lock, S. S. M et al. 2017. Molecular Simulation and Mathematical Modelling of Glass Transition Temperature Depression Induced by $\mathrm{CO}_{2}$ Plasticization in Polysulfone Membranes Molecular Simulation and Mathematical Modelling of Glass Transition Temperature Depression Induced by $\mathrm{CO}_{2}$. Material Science and Engineering.

[20] Lokhandwala, Kaaeid A, and Richard, W. Baker. 2008. Natural Gas Processing with Membranes. Industrial and Engineering Chemistry Research 1(19): 210921.

[21] Low, Ze-xian, Peter, M. Budd, Neil, B. Mckeown, and Darrell, A. Patterson. 2018. Gas Permeation Properties, Physical Aging, and Its Mitigation in High Free Volume Glassy Polymers. Chemical Reviews. 118(118): 5871-5911.

[22] Lu, H. T. et al. 2018. The Impact of Toluene and Xylene on the Performance of Cellulose Triacetate Membranes for Natural Gas Sweetening. Journal of Membrane Science. 555(January): 362-68.

[23] Miandoab, Ehsan Soroodan, Sandra, E. Kentish, and Colin, A. Scholes. 2021. Modelling Competitive Sorption and Plasticization of Glassy Polymeric Membranes Used in Biogas Upgrading. Journal of Membrane Science. 617(June 
2020):

118643.

https://doi.org/10.1016/j.memsci. 2020.118643.

[24] Minelli, Matteo, Stefano Oradei, Maurizio Fiorini, and Giulio C. Sarti. 2019. $\mathrm{CO}_{2}$ Plasticization Effect on Glassy Polymeric Membranes. Polymer.

[25] Sanders, David F. et al. 2013. Energy-Efficient Polymeric Gas Separation Membranes for a Sustainable Future: A Review. Polymer. 54(18): 4729-61.

[26] Scholes, Colin A., Sandra, E. Kentish, and Geoff, W. Stevens. 2009. Effects of Minor Components in Carbon Dioxide Capture Using Polymeric Gas Separation Membranes. Separation and Purification Reviews. 38(1): 1-44.

[27] Visser, T. 2006. Mixed Gas Plasticization Phenomena.

[28] Wypych, George, ed. 2017.
Handbook of Plasticizer. Third Edit. Toronto: ChemTec Publishing.

[29] Xia, Jianzhong, Tai Shung Chung, and D. R. Paul. 2014. Physical Aging and Carbon Dioxide Plasticization of Thin Polyimide Films in Mixed Gas Permeation. Journal of Membrane Science.

[30] Yong, Wai Fen, Tai-shung Shung Chung, Martin Weber, and Christian Maletzko. 2018. New Polyethersulfone (PESU) Hollow Fiber Membranes for $\mathrm{CO} 2$ Capture. Journal of Membrane Science 552(January): 305-14. https://doi.org/10.1016/j.memsci. 2018.02.008.

[31] Yong, Wai Fen, Kar Hui Andrel Kwek, Kuo Sung Liao, and Tai Shung Chung. 2015. Suppression of Aging and Plasticization in Highly Permeable Polymers. Polymer. 